\title{
Hypothalamic Myo-Inositol and Glucose concentrations, mood symptoms and fatigue in depression: a 3 Tesla chemical-shift-imaging in vivo proton magnetic resonance spectroscopy study
}

Pedraz-Petrozzi, Bruno ( $\sim$ bruno.pedraz@med.uni-giessen.de )

Cognitive Neuroscience at the Center of Psychiatry and Psychotherapy, Justus-Liebig University Giessen

Blecker, Carlo

Bender Institute of Neuroimaging, Justus-Liebig University Giessen

Sammer, Gebhard

Cognitive Neuroscience at the Center of Psychiatry and Psychotherapy, Justus-Liebig University Giessen

\section{Research Article}

Keywords: Depressive disorder, Fatigue, Proton Magnetic Resonance Spectroscopy, Hypothalamus, Glucose, Inositol

Posted Date: December 23rd, 2020

DOI: https://doi.org/10.21203/rs.3.rs-132378/v1

License: (c) (i) This work is licensed under a Creative Commons Attribution 4.0 International License. Read Full License 


\section{Abstract}

Background: Many studies have confirmed altered brain Myo-Inositol (mlns) and Glucose (Glc) concentrations in depression. Alterations in the mlnsconcentrations may represent a glial dysfunction and are associated mostly with depression symptoms. In comparison, alterations in the Glc-concentrations could represent impaired glucose metabolism. Despite this information, studies that assess these metabolites in the hypothalamus are missing. Therefore, this study aims to investigate mlns- and Glc-concentrations in the hypothalamus of participants with depression concerning mood symptoms and fatigue.

Methods: We performed an in vivo proton magnetic resonance spectroscopy - chemical shift imaging of the hypothalamus in 49 participants (25 with depression diagnosis or DE, 24 healthy controls or $\mathrm{HC}$ ), evaluating mIns- and Glc-concentrations. Concerning information about symptoms, we recollected information on depression symptomatology (BDI-FS TOT $_{\text {T }}$ ) and fatigue (FIS-D). For statistical analysis, we run generalized linear models by using the Poisson regression model with count data.

Results: The results showed reduced hypothalamic mlns-concentration for every DE participant with higher FIS-D and higher $\mathrm{BDI}_{\mathrm{FS}} \mathrm{TOT}_{\mathrm{T}}$ values $(\mathrm{OR}=0.022 ; p<$ 0.001 ; CI95 [0.004 to 0.09]). Besides, the results showed increased hypothalamic Glc-concentration for every participant of the DE group and with higher BDI$\mathrm{FS}_{\text {TOT }}$ values $(\mathrm{OR}=4.827 ; p=0.002 ; \mathrm{Cl} 95[1.826$ to 13.749$])$.

Conclusions: These results provide initial evidence of a glial dysfunction and impaired glucose metabolism in the hypothalamus associated with mood symptoms and fatigue. Future studies are needed to confirm these pathophysiologic mechanisms.

\section{Introduction}

Depression is defined as a mood disorder frequently related to increased functional impairment ${ }^{1,2}$. Although the knowledge of depression has evolved substantially in the last decades, the origins and physiopathology of this mood disorder remain to date unknown ${ }^{3}$.

Over the last years, scientists have postulated an alteration of the hypothalamus (HYP) in depression ${ }^{4,5}$. In this matter, some models support this theory on depressive disorders postulating a chronic dysregulation of the HYP ${ }^{4,6}$. For instance, the result of two studies showed that HYP overactivation correlates positively with higher relapse of depression and the number of depressive episodes over time ${ }^{4,7-9}$. Besides, other studies showed that this dysregulation of the HYP relies on hyperactive $\mathrm{CRH}$ neurons. These are crucial for the induction of depressive symptomatology in some groups of patients with depression ${ }^{4,10}$.

The HYP dysregulation also involves metabolic processes, which could be assessed using in vivo proton magnetic resonance spectroscopy ( $\left.{ }^{1} \mathrm{H}-\mathrm{MRS}\right){ }^{11,12}$. The latter enables a non-invasive detection by using strong magnetic fields of various hypothalamic metabolites concentrations, including Myo-Inositol and Glucose ${ }^{13}$. The first molecule, Myolnositol ( $\mathrm{mlns}$ ), is a simple sugar that serves as an intracellular second-messenger ${ }^{14}$. In the central nervous system (CNS), $\mathrm{mlns}$ is synthesized in glial cells, predominantly in the astrocytes ${ }^{15}$. The second molecule, Glucose (Glc), is another important molecule in the CNS, which is used as the primary source of energy. This molecule is also involved in the glutamine-glutamate-metabolism in the astrocytes ${ }^{16}$.

There are few studies where mlns is involved in the dysregulation process of the HYP in depression. For example, the study of de Rezende et al. (2018) found out that mIns-concentrations are positively correlated with HYP overactivation in postpartum depression ${ }^{17}$. On the contrary, the study of Rui Yang et al. (2009) showed significantly reduced hypothalamic mlns-concentrations in participants with the first-episode depression ${ }^{18}$. Since there are few but heterogeneous results, more studies are needed to clarify the role of mIns-concentration on the HYP dysregulation in depression. In contrast to mIns and depression, there are no studies that evaluate hypothalamic Glc-concentrations in depression by using ${ }^{1} \mathrm{H}-\mathrm{MRS}$. Only one study by Ernst et al. (2017) demonstrates higher Glcconcentrations in depression by using ${ }^{1} \mathrm{H}-\mathrm{MRS}^{3}$. However, this study evaluated only the pregenual anterior cingulate cortex (PACC), which is not part of the hypothalamic structure. Glc is the primary cerebral energy substrate and a metabolite for glutamate metabolism; its increased concentration could relate to higher activity and metabolism ${ }^{3}$. For this reason, more studies are needed to define the relationship between GIc-concentrations and HYP dysregulation in depression.

To this end, this study has two aims. Firstly, to investigate the levels of mlns and Glc in the HYP in participants with a depressive episode. Lastly, to investigate the levels of these metabolites in relationship with depression symptoms. For this last aim, we included fatigue as a symptom in this study since it is also related to HYP dysregulation and is frequent in depression ${ }^{19}$.

Following the previous findings, we hypothesize that the concentrations of both metabolites in the HYP will differ between participants with depression and healthy controls as a reflex for the dysregulation theory. Moreover, we hypothesize that both metabolites' concentrations will associate, in this case, with levels of depression symptoms, like fatigue.

\section{Materials And Methods}

This study's information comes from a database of a bigger project that involved participants with depression as well as healthy community controls, and that encompasses magnetic resonance imaging (MRI), functional magnetic resonance imaging (fMRI), diffusion-tension-imaging (DTI), and in vivo proton magnetic resonance spectroscopy ( $\left.{ }^{1} \mathrm{H}-\mathrm{MRS}\right)$. Some results of this project were published elsewhere by Pedraz-Petrozzi et al. ${ }^{24}$.

\subsection{Study participants}

Between June and September 2019, 49 participants were recruited for this study. Two groups were defined: a group of participants with depression (DE; $\mathrm{n}$ = 24) and another one with healthy community controls $(\mathrm{HC} ; \mathrm{n}=25)$. Both of them were matched according to age and gender. General socio-demographic 
characteristics of both groups are described in the chapter 4.1 .

Participants for the DE group were included if they fulfilled the criteria for a depressive episode of the tenth version of the International Classification of Diseases (ICD-10) and did not present any comorbid psychotic episode. Additionally, participants with other psychiatric disorders (e.g., personality disorders) were also included as long as a depressive episode predominated for the past six months.

Insufficient knowledge of the German language and severe somatic restrictions (e.g., impaired vision or hearing) were considered in this study as exclusion criteria. Also, participants older than 65 or younger than 18 years were excluded from the study. Except for depressive episodes in the DE group, any medical disease was considered as an exclusion criterion. Finally, participants with contraindications for MRI examination (such as ferromagnetic medical devices) were excluded from this study.

\subsection{Ethics}

This study was approved by the ethical commission from the Faculty of Medicine of the Justus Liebig University - Giessen. All procedures were performed according to the Helsinki Declaration and the American Psychology Association standards. Participants or their authorized representatives were informed of the study procedures and signed an informed consent document before participation.

\subsection{Depression: diagnosis and severity}

A depressive episode was defined following the ICD-10 throughout a structural interview ${ }^{20,21}$. The latter was carried out by clinical experts from the Department of Psychiatry of the University Hospital Giessen.

To evaluate the severity of depressive symptoms, the German version of the Beck Depression Inventory - Fast Screening (BDI-FS) was handed out to each participant ${ }^{20,22}$. This instrument delivers values between 0 and 21 points. A higher value denotes a higher depression severity. The BDI-FS was validated in Germany and showed good internal consistency (Cronbach's $a=0.84)$ and convergent validity with other depression instruments $(\mathrm{PHQ}-9$ : $r=0.67)$. Categories for depressive episodes were defined in the instrument manual by using the total score as follows: minimal ( 0 to 3 points), mild (4 to 8 points), moderate ( 9 to 12 points), and severe (13 to 21 points) ${ }^{20}$.

\subsection{Fatigue}

Fatigue was defined clinically and measured using the Fatigue Impact Scale - German Version (FIS-D), which was also validated in Germany ${ }^{23}$.

The FIS-D has the benefit of evaluating the fatigue impact based on the health-related Quality of Life. This scale contained three different dimensions: psychosocial, somatic, and cognitive dimensions. The total score of the FIS-D was obtained by adding the points for each question (40 questions, highest score $=160)$. As defined in the scale manual, the cut-off value for the FIS-D total score was 40.

\subsection{T in vivo magnetic resonance spectroscopy of the hypothalamus: chemical shift imaging}

\subsubsection{Data acquisition}

Participants underwent a ${ }^{1} \mathrm{H}-\mathrm{MRS}$ imaging protocol using a SIEMENS MAGNETROM Prisma 3.0 Tesla MRI scanner with a 64-channel head coil (Bender Institute for Neuroimaging, Faculty of Psychology, Justus-Liebig University, Giessen). This study followed the data acquisition protocol of Pedraz-Petrozzi et al., published elsewhere ${ }^{24}$.

This ${ }^{1} \mathrm{H}$-MRS imaging protocol included a T1-weighted magnetization prepared rapid acquisition gradient echo (MPRAGE) image in sagittal position, a ${ }^{1} \mathrm{H}$ MRS localizer protocol for chemical shift imaging (CSI), and a CSI with spin-echo sequence (SE) ${ }^{1} \mathrm{H}$-MRS. Figure 1 shows the region of interest (ROI) used for this study ${ }^{24}$.

MPRAGE parameters were set to TR/TE $=1580 \mathrm{~ms} / 2.3 \mathrm{~ms}, 176$ slices, slice thickness $=0.94 \mathrm{~mm}$, field of view $($ FoV $)=240 \mathrm{~mm} \times 240 \mathrm{~mm}, \mathrm{matrix}$ size $=256 \mathrm{x}$ 256 voxels, duration $=4$ minutes and 29 seconds $^{24}$. For running the ${ }^{1} \mathrm{H}$-MRS localizer, images from the T1-weighted MPRAGE were used to locate the slice containing the hypothalamus (reference structures: thalamus, mammillary bodies, inferior and superior colliculi, pituitary gland, and fornix) ${ }^{24}$. The parameters were set to TR/TE $=6000 \mathrm{~ms} / 115 \mathrm{~ms}, 1$ slice group, 11 slices, FoV $=220 \mathrm{~mm} \times 220 \mathrm{~mm}$, slice thickness $=5 \mathrm{~mm}$, distortion factor $=0 \%$, duration $=1 \mathrm{minute}$ and 14 seconds $^{24}$. The parameters for the CSI with SE ${ }^{1} \mathrm{H}$-MRS protocol were set to TR/TE $=1700 \mathrm{~ms} / 30 \mathrm{~ms}, \mathrm{FoV}=160 \mathrm{~mm} \times 160 \mathrm{~mm}, \mathrm{voxel}$ size $=10 \mathrm{~mm} \times$ $10 \mathrm{~mm} \times 15 \mathrm{~mm}$, volume of interest (VOI) $=80 \mathrm{~mm} \times 80 \mathrm{~mm}$, number of signal averaging $=3$, duration $=6$ minutes and 53 seconds 24 . In this case for CSI, the VOI is divided into 64 voxels (in this study, volume for each voxel: $10 \mathrm{~mm} \times 10 \mathrm{~mm} \times 15 \mathrm{~mm}$ ) ${ }^{24}$. The analyzed voxels for this study are marked in figures 1 and 2.

\subsubsection{Imaging processing}

The imaging processing methods were followed from the study of Pedraz-Petrozzi et al., published elsewhere ${ }^{24}$.

Two blinded researchers (neuroimaging scientists) evaluated the data obtained from the ${ }^{1} \mathrm{H}-\mathrm{MRS}^{24}$. The data analysis was carried out on the selected layer with TARQUIN (Totally Automatic Robust Quantitation in $N M R^{25}$ ) version 4.3.1. This software was selected based on positive reports in the literature ${ }^{26,27}$. For this study, Myo-Inositol (mIns) and Glucose (Glc) from the water-suppressed CSI were quantified using SE ${ }^{1} \mathrm{H}-\mathrm{MRS}$ data ${ }^{24}$. Total creatinine (tCr) values were also calculated and used as a reference signal for the analysis ${ }^{24}$. 
The ${ }^{1} \mathrm{H}$-MRS data obtained for the hypothalamic region could contain many voxels for each participant ${ }^{24}$. For this reason, the voxels with a signal-to-noise ratio (SNR) for the metabolites and a reference signal $>3$ were selected ${ }^{24}$. Hypothalamic voxels with an SNR $<3$ for these metabolites were discarded. The metabolite concentrations ( $\mathrm{mlns}, \mathrm{Glc}$ ) were calculated for these selected voxels and divided by a reference metabolite ( $\mathrm{tCr}$ ). The methodological

considerations for ${ }^{1} \mathrm{H}$-MRS data analysis, particularly for determining the metabolite concentrations, have been published elsewhere in the literature ${ }^{28}$. Finally, the voxel with the highest concentration was selected for each participant and each of the metabolites ( $\mathrm{mlns} / \mathrm{tCr}$, $\mathrm{Glc} / \mathrm{tCr}$ ) and fed into the statistical analysis ${ }^{24}$.

\subsection{Statistical analysis}

Statistical analyses were performed using SPSS version 26.0 (International Business Machines Corporation, New York, United States of America) and the toolbox GAMLj ${ }^{29}$ of the statistics package jamovi $1.2 \cdot 5 \cdot 0^{30}$.

\subsubsection{Descriptive data}

Measures of central tendency were used to describe the data and their distribution. Also, skewness and kurtosis were calculated to determine the asymmetry of the data concerning the normal distribution. The Shapiro-Wilk test was used to test deviation from the normality distribution. An approximation to the normal distribution is given with a p-value greater than 0.05 .

Categorical variables were specified with numbers and, in some cases, quotient values. For obtaining results with two decimals, data were rounded to the next decimal.

All data were organized in tables to improve readability. General descriptive information, which included psychometric data, is mentioned in chapter 4.1 . Descriptive information regarding hypothalamic metabolites is also mentioned in chapter 4.1.

\subsubsection{Hypothalamic metabolites' data}

The data obtained from the MRS (mlns/tCr, Glc/tCr) showed positively skewed, non-Gaussian distributions with a few large values. This dataset, which was expressed in parts per million (ppm), corresponded to count data that is discretely distributed. In this case, the Poisson distribution is suitable for such count data. Poisson distributions can handle positively skewed data and a larger kurtosis, indicating the existence of some larger values.

Accordingly, generalized linear models were calculated. The model type was adapted to the Poisson distribution. The hypothalamic metabolites mIns/tCr or

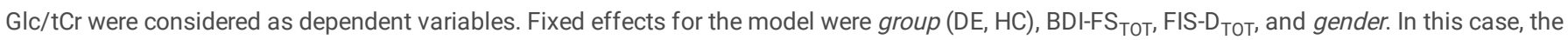
psychometric data (BDI-FS TOT, FIS-D $_{\text {TOT }}$ ) were dichotomized using the median split method, defining two categories (lower and higher) and introduced in the model as fixed effects. BMI and age were considered as covariates for this model to control the possible effects of these variables.

The models were calculated separately for Glc/tCr and mlns/tCr. In the modeling process, it was found that the variables age, gender, smoking behavior, and medication intake contributed minimally to the explanation of the variance. Therefore, they were removed from the final model. The proportion of error reduction was greater with this model than with a general linear Gaussian model (R-square 0.33: 0.22).

For these models, values were flagged 'significant' if the Bonferroni corrected ( $P_{\text {BONF }}$ ) two-tailed p-value was $P_{\text {BONF }} \leq 0.025$ (for both metabolites mlns and Glc: $\mathrm{p} / 2$ = $\left.\mathrm{P}_{\mathrm{BONF}}\right)$.

\section{Results}

\subsection{General characteristics of the sample}

The general characteristics of the 49 participants, including the hypothalamus metabolites' concentrations, are described in table 1. Both groups had the same number of female participants (HC: 15 female participants; DE: 15 female participants). Age did not differ between both groups (U = 243; $p=0.257$ ), as well as $\mathrm{BMI}(\mathrm{U}=299 ; p=0.992)$.

For the HC group, only 5 of 25 participants got higher FIS-D values. In contrast, the DE group got 20 of 24 participants with higher FIS-D values. Similar results

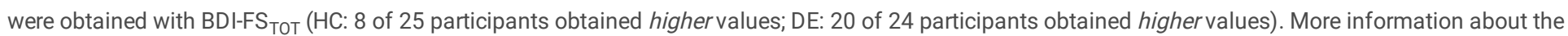
values following Median Split Method is represented in table 2. 


\begin{tabular}{|c|c|c|c|c|c|c|c|}
\hline & $\begin{array}{l}\text { Group } \\
\text { Status }\end{array}$ & Age & BMI & $\mathrm{BDI}^{-F S_{\text {TOT }}}$ & FIS-D & $\mathrm{mlns} / \mathrm{tCr}$ & $\mathrm{Glc} / \mathrm{tCr}$ \\
\hline \multirow[t]{2}{*}{$\mathrm{N}$} & $\mathrm{HC}$ & 25 & 25 & 25 & 25 & 25 & 25 \\
\hline & $\mathrm{DE}$ & 24 & 24 & 24 & 24 & 24 & 24 \\
\hline \multirow[t]{2}{*}{ Mean \pm SD } & $\mathrm{HC}$ & $26.80 \pm 8.73$ & $24.90 \pm 5.86$ & $1.80 \pm 2.16$ & $28.20 \pm 17.70$ & $7.23 \pm 15.00$ & $2.39 \pm 1.61$ \\
\hline & $\mathrm{DE}$ & $31.90 \pm 12.80$ & $24.00 \pm 4.17$ & $7.25 \pm 5.17$ & $74.10 \pm 32.60$ & $12.00 \pm 27.10$ & $5.37 \pm 5.70$ \\
\hline \multirow[t]{2}{*}{ Median (Q3 - Q1) } & $\mathrm{HC}$ & $\begin{array}{l}25.00(29.00- \\
22.00)\end{array}$ & $\begin{array}{l}22.30(25.30- \\
21.40)\end{array}$ & $\begin{array}{l}1.00(3.00- \\
0.00)\end{array}$ & $\begin{array}{l}27.00(41.00- \\
13.00)\end{array}$ & $\begin{array}{l}0.36(4.21- \\
0.00)\end{array}$ & $\begin{array}{l}1.88(3.51- \\
1.31)\end{array}$ \\
\hline & $\mathrm{DE}$ & $\begin{array}{l}26.50(36.30- \\
22.00)\end{array}$ & $\begin{array}{l}23.40(27.40- \\
25.30)\end{array}$ & $\begin{array}{l}7.00(10.00- \\
3.00)\end{array}$ & $\begin{array}{l}82.00(95.30- \\
49.00)\end{array}$ & $\begin{array}{l}3.40(12.10- \\
0.20)\end{array}$ & $\begin{array}{l}3.45(7.05- \\
1.65)\end{array}$ \\
\hline \multirow[t]{2}{*}{ Skewness } & $\mathrm{HC}$ & 3.42 & 1.92 & 1.76 & 0.27 & 2.59 & 0.41 \\
\hline & $\mathrm{DE}$ & 1.30 & 0.66 & 0.53 & 0.10 & 4.16 & 1.96 \\
\hline \multirow[t]{2}{*}{ Kurtosis } & $\mathrm{HC}$ & 14.40 & 2.82 & 3.85 & -0.85 & 6.61 & -0.68 \\
\hline & $\mathrm{DE}$ & 0.73 & 0.31 & -0.64 & -0.73 & 18.8 & 3.85 \\
\hline \multirow[t]{2}{*}{ Shapiro-Wilk's W } & $\mathrm{HC}$ & 0.642 & 0.720 & 0.786 & 0.961 & 0.56 & 0.94 \\
\hline & $\mathrm{DE}$ & 0.822 & 0.959 & 0.930 & 0.963 & 0.45 & 0.77 \\
\hline \multirow{2}{*}{$\begin{array}{l}\text { Shapiro-Wilk's p- } \\
\text { value }\end{array}$} & $\mathrm{HC}$ & $<0.001$ & $<0.001$ & $<0.001$ & 0.43 & $<0.001$ & 0.16 \\
\hline & $\mathrm{DE}$ & $<0.001$ & 0.43 & 0.10 & 0.50 & $<0.001$ & $<0.001$ \\
\hline
\end{tabular}

Table 1: General characteristics of the participants. Abbreviations - BMl: Body mass index (in kg/m2); BDI-FS Inventory fast screening; FIS-D: Fatigue Impact Scale, German Version; mIns/tCr: Myo-Inositol concentration; Glc/tCr: Glucose concentration; HC: healthy controls; DE: participants with depressive episode; SD: Standard deviation; Q3: $3^{\text {rd }}$ quartile; Q1: $1^{\text {st }}$ quartile.

\begin{tabular}{|c|c|c|c|c|}
\hline & \multicolumn{4}{|c|}{ BDI-FS $_{\text {Tот }}$} \\
\hline & Group Status & Higher & Lower & Total \\
\hline \multirow[t]{3}{*}{$\mathrm{N}$} & $\mathrm{HC}$ & 8 & 17 & 25 \\
\hline & $\mathrm{DE}$ & 20 & 4 & 24 \\
\hline & Total & 28 & 21 & 49 \\
\hline \multicolumn{5}{|c|}{ FIS-D } \\
\hline & Group Status & Higher & Lower & Total \\
\hline \multirow[t]{3}{*}{$\mathrm{N}$} & $\mathrm{HC}$ & 5 & 20 & 25 \\
\hline & $\mathrm{DE}$ & 20 & 4 & 24 \\
\hline & Total & 25 & 24 & 49 \\
\hline
\end{tabular}

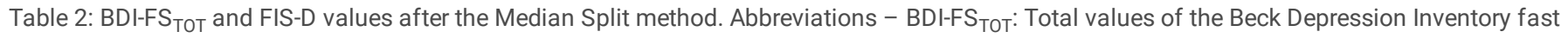
screening; FIS-D: Fatigue Impact Scale, German Version; HC: healthy controls; DE: participants with depressive episode.

\subsection{Myo-Inositol}

Regarding mlns-concentrations, Poisson regression was computed to predict the hypothalamic mlns-concentrations based on the group status (DE or HC), the

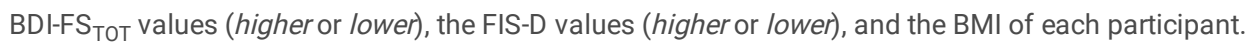

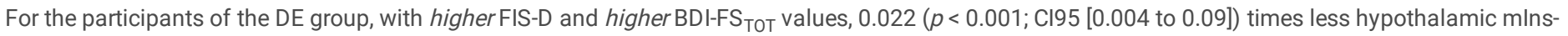
concentration was obtained. Additionally, this predictor (i.e., Status $\times$ BDI-FS TOT $\times$ FIS-D) fitted in the Poisson model type $(\mathrm{X} 2=35.44 ; \mathrm{df}=1 ; p<0.001)$. Figure 3 represents graphical information about hypothalamic mIns-concentration and group status by higherFIS-D values. The information in the graphic was divided for higher and lower BDI-FS ${ }_{\text {TOT }}$ values. 
Additionally, BMI predicted positively the mIns-concentrations in this model. This means that for every increase of $\mathrm{BMI}, 1.140(p<0.001 ; \mathrm{Cl} 95$ [1.120 to 1.157$])$ times more hypothalamic mIns-concentration was obtained. BMI fitted also in the Poisson model type $(\chi 2=253.65 ; \mathrm{df}=1 ; p<0.001)$.

\subsection{Glucose}

In the case of the Glc-concentrations, Poisson regression was also run to predict the Glc-concentrations in the hypothalamus based on the group status (DE or $\mathrm{HC}$ ), the BDI-FS

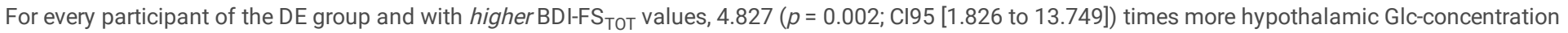
was obtained. Additionally, this predictor (i.e., Status $x$ BDI-FS $\left.S_{T O T}\right)$ fitted in the Poisson model type $(\chi 2=10.28 ; d f=1 ; p=0.001)$. Figure 4 represents graphical

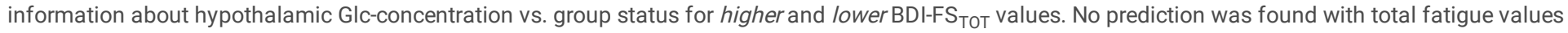
and hypothalamic Glc-concentration.

Additionally, BMI predicted negatively the Glc-concentrations in this model. This means that for every increase of $\mathrm{BMI}, 0.936(p=0.001 ; \mathrm{Cl} 95$ [0.897 to 0.973$])$ times less hypothalamic Glc-concentration was obtained. In case of hypothalamic Glc-concentrations, BMI fitted also in the Poisson model type $(X 2=12.05$; df $=1 ; p<0.001)$.

\section{Discussion}

This study aimed to investigate the levels of mlns and Glc in the HYP by participants with depressive episodes, involving psychometric evaluations for depression symptomatology and fatigue.

The results of this study showed reduced hypothalamic mlns-concentrations in participants with depression with higher symptom scores (i.e., higher BDI$\mathrm{FS}_{\text {TOT }}$ and higher FIS-D). In the brain tissue, mIns could be found, under physiological circumstances, in higher concentrations. This sugar molecule plays an essential role by making other neurotransmitters bind to their corresponding receptors. Besides, mlns is the structural basis for many secondary messengers, such as phosphatidylinositol (PI). Another critical function of mlns is the contribution to glial and astrocytic osmoregulatory functioning ${ }^{14,31,32}$.

Different studies have shown a negative correlation between brain mlns-concentration and mood symptomatology in depression ${ }^{14,33,34}$. Studies with ${ }^{1} \mathrm{H}-\mathrm{MRS}$ and depression reported decreased mIns-concentration in other brain regions, such as the anterior cingulate cortex and the prefrontal region ${ }^{14,35-37}$. Moreover, mlns-concentrations not only correlated negatively with mood symptomatology but also with daytime sleepiness and insomnia symptoms ${ }^{38}$. On the contrary, psychosocial functioning and total sleep time showed positive correlations with mlns-concentrations ${ }^{38}$.

This lower mlns-concentration could signalize two different pathophysiological phenomena. On the one hand, that there might be a disturbing second messenger system in depression ${ }^{38}$. On the other hand, there might also be a possible glial or astrocytic dysfunction in depression ${ }^{14,35,36}$.

Our study showed reduced mins-concentrations in the hypothalamus in participants with depression and higher symptoms. These findings could also correspond to glial damage in the hypothalamus. To date, there is only one study that reported reduced mlns-concentration in the hypothalamus and thalamus in depression ${ }^{18}$.

Noteworthy were the findings with total fatigue scores since fatigue is caused by HYP overactivation and is a consequence of chronic stress ${ }^{19}$. The latter showed to also contribute to the glial disfunction. For instance, in two experimental mice models, chronic stress showed a reduction in oligodendrocytes' proliferation (glia, astrocytes) ${ }^{39-41}$.

Finally, our study showed differences with de Rezende et al., which reported positive correlations between mIns-concentrations and HYP overactivation ${ }^{17}$. Firstly, the mIns-concentrations were obtained from the anterior cingulate cortex (ACC). Our study reported mlns-concentrations measured directly from the HYP, as also published in other studies ${ }^{24,42,43}$. Secondly, the selected participants were postpartum depressive patients. Our study participants were chosen without any additional medical condition, including pregnancy and puerperal patients. Finally, the definition of the HYP overactivation was the diurnal variation of cortisol. On the contrary, our study measured the mIns-concentrations directly from the HYP and defined fatigue as HYP overactivation ${ }^{19}$.

Regarding Glc-concentration, this study showed increased hypothalamic Glc-concentration in participants with depression with higher symptom scores (i.e., higher $\mathrm{BDI}^{-\mathrm{FS}_{\mathrm{TOT}}}$ ). To the best of our knowledge, this is the first study to report increased hypothalamic Glc-concentrations in participants with depression with higher mood symptom scores by using ${ }^{1} \mathrm{H}$-MRS. The study of Ernst et al. was the first study to report Glc-concentration differences between participants with depression and healthy controls ${ }^{3}$. However, Ernst et al. assessed the pregenual anterior cingulate cortex through ${ }^{1} \mathrm{H}-\mathrm{MRS}^{3}$. On the contrary, this study also reported increased Glc-concentrations, but the ROI was the HYP.

Glucose is the principal cerebral energy substrate in the brain, and it is supplied via circulation to the brain ${ }^{3}$. In this case, most of the hypothalamus' blood supply is given by the anterior cerebral artery and branches from the Willis circle. The study of Mayberg et al. reported that participants with depression showed significant cerebral blood flow reductions in the hypothalamus after deep brain stimulation treatment ${ }^{44}$. Another explanatory factor that could help to understand the findings of our study is the role of brain insulin resistance in depression ${ }^{45}$. Insulin is a hormone that increases the cellular intake of peripheral glucose ${ }^{46}$. In the last decades, it was demonstrated that there are also insulin receptors distributed in the CNS, and these could also have multiple effects on cognition and behavior ${ }^{45}$. Recently, an experimental study with rats showed that the downregulation of hypothalamic brain insulin receptors contributed to

Page 6/12 
depression-like symptoms like anhedonia and a decrease in active behaviors ${ }^{47}$. Benedict et al. showed positive effects on mood (increased self-confidence, enhanced mood, and reduced anger) after intranasal insulin administration in healthy subjects. These changes were attributed to modifying the hippocampus activity and some hypothalamus regions (i.e., paraventricular nucleus and ventromedial area) ${ }^{48}$.

Our study results are consistent with the mentioned research about the theory of brain insulin resistance $45,47,48$. In this case, we suggest that the increase of hypothalamic Glc-concentration in depression may be related to a possible brain insulin activity failure in this region. Nevertheless, more studies are needed to show that the increase of hypothalamic Glc-concentration is due to insulin resistance in this region.

Although they did not correspond to this study's main aims, BMI findings were also reported here. Higher BMI values are associated with reduced hypothalamic Glc-concentration and increased mIns-concentration. To date, there are no human studies that evaluated BMI with mIns- or Glc-concentrations in the HYP. However, two experimental animal studies have found that increased hypothalamic mlns-concentration is correlated with obesity and appetite behavior ${ }^{49,50}$. Our findings obtained between BMI and hypothalamic Glc-concentration differ with the systematic review of Lizarbe et al., which reported a high glycemic concentration in brain tissue of animal models with high-fat diet consumption and obesity by using MRS ${ }^{51}$. One possibility that could explain these differences is that our sample size did not include clinically obese subjects or metabolic syndrome. Before starting the study, the authors of this study checked if the participants met the exclusion criteria. Therefore, all subjects with diabetes mellitus or metabolic syndrome were excluded from this study.

Although this study showed relevant findings, it is important to mention some limitations of our research. Firstly, the sample size could restrict the generalization of the obtained results. Nevertheless, we calculated that the estimated power (1- $\beta$ ) for our total sample size of 49 participants is 0.98 . The power for our sample size is above the accepted minimum power threshold $(1-\beta=0.80)$, indicating that the sample size for this study design is sufficient to achieve the study goals. Secondly, we have included more women than men in the study. However, the greater number of female participants coincide with the statistics of depression in Germany (female > male) ${ }^{52,53}$. Finally, there were limitations regarding the ${ }^{1} \mathrm{H}$-MRS with CSI. Although all extracted voxels come from a brain slice that contained the HYP, there is a theoretical possibility that other neighboring structures (i.e., thalamus, corpus callosum, hippocampus, etc.) may have lightly contributed to the signals attributed to the HYP. As far as we know, the method is currently one of the best for detecting non-invasive HYP metabolite levels ${ }^{24,42,43}$

In conclusion, high mood symptoms and fatigue in depression are associated with reduced hypothalamic mlns-concentration. The severity of depressive symptoms explained in this case, the reduced amount of mlns-concentration in the hypothalamus. The following suggests that in depression, a glial or astrocyte dysfunction in the hypothalamus may be present. Besides, high mood symptoms but not fatigue is associated with augmented hypothalamic Glcconcentrations. The latter suggests that a brain insulin resistance mechanism in the neural tissue could augment hypothalamic glucose in depression. However, future studies are needed to confirm the relationship between insulin resistance and increased Glc-concentration in the HYP by depression.

\section{Declarations}

Non-financial competing interests: The authors declare that they have no competing interests.

Financial competing interests

- Funding: This study is part of a project entitled: "Correlations between systemic inflammation, brain endophenotypes and fatigue in participants with depression". This project has received a financial support from the German Exchange Academic Program (DAAD). This program included also the financial support of the project, that involved mainly material expenses and participants' expenses for participation. Additionally, the authors of this project contacted the owner of the "Immunität und Seele" foundation (Prof. Dr. Norbert Müller), that awarded this project and supported financially. Both supporters have no role in the design of the study, data collection, analysis and interpretation of results.

All other authors have no conflicts to declare.

Employment: The authors of this study were not hired by any organization that may gain or lose financially through this publication.

Personal financial interests: With exception of point "Funding", for this study the authors did not received any compensation (monetary, shares, etc.) from companies, consultation fees or other forms of remuneration from any organizations.

Acknowledgements: This study was supported financially by the German academic exchange program (DAAD) and by the foundation "Immunität und Seele" (Prof. Norbert Müller). The authors would like to thank the neuroimaging team of the Bender Institute of Neuroimaging (BION) from the Psychology and Sports Science faculty - Justus Liebig University (Giessen).

\section{Bibliography}

1. World Health Organization. Depression and Other Common Mental Disorders. Institutes Heal. Natl. (2017). doi:CC BY-NC-SA 3.0 IGO

2. Wang, J. et al. Prevalence of depression and depressive symptoms among outpatients: a systematic review and meta-analysis. BMJ Open 7, e017173 (2017).

3. Ernst, J. et al. Increased pregenual anterior cingulate glucose and lactate concentrations in major depressive disorder. Mol. Psychiatry 22, 113-119 (2017). 
4. Varghese, F. P. \& Brown, E. S. The Hypothalamic-Pituitary-Adrenal Axis in Major Depressive Disorder. Prim. Care Companion J. Clin. Psychiatry 03, 151155 (2001).

5. Holsboer, F. \& Barden, N. Antidepressants and hypothalamic-pituitary-adrenocortical regulation. Endocr. Rev. 17, 187-205 (1996).

6. Menke, A. Is the HPA Axis as Target for Depression Outdated, or Is There a New Hope? Front. Psychiatry 10, 1-8 (2019).

7. Lenox, R. H., Peyser, J. M., Rothschild, B., Shipley, J. \& Weaver, L. Failure to normalize the dexamethasone suppression test: Association with length of illness. Biol. Psychiatry 20, 333-337 (1985).

8. Yerevanian, B. I., Feusner, J. D., Koek, R. J. \& Mintz, J. The dexamethasone suppression test as a predictor of suicidal behavior in unipolar depression. J. Affect. Disord. (2004). doi:10.1016/j.jad.2004.08.009

9. Yerevanian, B. I., Privitera, M. R., Milanese, E., Sagi, E. A. \& Russotto, J. C. The dexamethasone suppression test during recurrent major depressive episodes. Biol. Psychiatry (1984).

10. Rama Krishnan, K. R. et al. Pituitary size in depression. J. Clin. Endocrinol. Metab. (1991). doi:10.1210/jcem-72-2-256

11. Hasler, G. \& Northoff, G. Discovering imaging endophenotypes for major depression. Mol. Psychiatry 16, 604-619 (2011).

12. Peterson, B. S. \& Weissman, M. M. A Brain-Based Endophenotype for Major Depressive Disorder. Annu. Rev. Med. 62, 461-474 (2011).

13. Edelman, R. R. The History of MR Imaging as Seen through the Pages of Radiology. Radiology 273, S181-S200 (2014).

14. Chiappelli, J. et al. Evaluation of Myo-Inositol as a Potential Biomarker for Depression in Schizophrenia. Neuropsychopharmacology 40, 2157-2164 (2015).

15. Hamsini, B. C., Reddy, B. N., Neelakantan, S. \& Kumaran, S. P. Clinical Application of MR Spectroscopy in Identifying Biochemical Composition of the Intracranial Pathologies. in GABA And Glutamate - New Developments In Neurotransmission Research i, 13 (InTech, 2018).

16. Conti, F. Capitulo 11 - Sistema nervioso: componentes y organización. in Fisiología Médica (Fiorenzo Conti, compilador) 250-277 (2010).

17. de Rezende, M. G. et al. Correlations between changes in the hypothalamic-pituitary-adrenal axis and neurochemistry of the anterior cingulate gyrus in postpartum depression. J. Affect. Disord. 239, 274-281 (2018).

18. Yang, R. et al. [Proton magnetic resonance spectroscopy of the thalamus and hypothalamus in patients with first-episode depression]. Nan Fang Yi Ke Da Xue Xue Bao (2009).

19. Henn, F. A. \& Vollmayr, B. Stress models of depression: Forming genetically vulnerable strains. Neuroscience and Biobehavioral Reviews (2005). doi:10.1016/j.neubiorev.2005.03.019

20. Pedraz-Petrozzi, B., Neumann, E. \& Sammer, G. Pro-inflammatory markers and fatigue in patients with depression: A case-control study. Sci. Rep. 10, 1-12 (2020).

21. World Health Organization. The ICD-10 classification of mental and behavioural disorders: Diagnostic criteria for research. The ICD-10 classification of mental and behavioural disorders: Diagnostic criteria for research (1993).

22. Kliem, S., Mößle, T., Zenger, M. \& Brähler, E. Reliability and validity of the beck depression inventory-fast screen for medical patients in the general German population. J. Affect. Disord. (2014). doi:10.1016/j.jad.2013.11.024

23. Häuser, W., Almouhtasseb, R., Muthny, F. A. \& Grandt, D. Validierung der Deutschen Version der Fatigue Impact Scale FIS-D. Z. Gastroenterol. 41, $973-982$ (2003).

24. Pedraz-Petrozzi, B., Blecker, C., Neumann, E. \& Sammer, G. The secret behind peripheral inflammation and depression: The hypothalamus (PREPRINT). Res. Sq. (2020). doi:10.21203/rs.3.rs-53606/v1

25. Wilson, M., Reynolds, G., Kauppinen, R. A., Arvanitis, T. N. \& Peet, A. C. A constrained least-squares approach to the automated quantitation of in vivo ${ }^{1}$ H magnetic resonance spectroscopy data. Magn. Reson. Med. 65, 1-12 (2011).

26. Scott, J., Underwood, J., Garvey, L. J., Mora-Peris, B. \& Winston, A. A comparison of two post-processing analysis methods to quantify cerebral metabolites measured via proton magnetic resonance spectroscopy in HIV disease. Br. J. Radiol. 89, 20150979 (2016).

27. Tsougos, I. Advanced MR Neuroimaging: From Theory to Clinical Practice. (CRC Press, 2017).

28. Barker, P. B., Bonekamp, D., Riedy, G. \& Smith, M. Quantitation of NAA in the Brain by Magnetic Resonance Spectroscopy. in N-Acetylaspartate (eds. Moffett, J. R., Tieman, S. B., Weinberger, D. R., Coyle, J. T. \& Namboodiri, A. M. A.) 183-197 (Springer US, 2006). doi:10.1007/0-387-30172-0_13

29. Gallucci, M. GAMLJ - General Analyses for Linear Models. (2019). Available at: https://www.jamovi.org/library.html. (Accessed: 22nd June 2020)

30. Love, J. et al. The jamovi project. (2020). Available at: https://www.jamovi.org. (Accessed: 22nd June 2020)

31. Strange, K. Regulation of solute and water balance and cell volume in the central nervous system. J. Am. Soc. Nephrol. 3, 12-27 (1992).

32. Fisher, S. K., Novak, J. E. \& Agranoff, B. W. Inositol and higher inositol phosphates in neural tissues: homeostasis, metabolism and functional significance. J. Neurochem. 82, 736-54 (2002).

33. Ongur, D., Drevets, W. C. \& Price, J. L. Glial reduction in the subgenual prefrontal cortex in mood disorders. Proc. Natl. Acad. Sci. 95, 13290-13295 (1998).

34. Hamidi, M., Drevets, W. C. \& Price, J. L. Glial reduction in amygdala in major depressive disorder is due to oligodendrocytes. Biol. Psychiatry 55, 563-569 (2004).

35. Coupland, N. J. et al. Decreased prefrontal myo-inositol in major depressive disorder. Biol. Psychiatry (2005). doi:10.1016/j.biopsych.2005.02.027

36. Chen, L.-P., Dai, H.-Y., Dai, Z.-Z., Xu, C.-T. \& Wu, R.-H. Anterior cingulate cortex and cerebellar hemisphere neurometabolite changes in depression treatment: A $1 \mathrm{H}$ magnetic resonance spectroscopy study. Psychiatry Clin. Neurosci. 68, 357-364 (2014).

37. Frey, R. et al. Myo-inositol in depressive and healthy subjects determined by frontal $1 \mathrm{H}$-magnetic resonance spectroscopy at 1.5 tesla. J. Psychiatr. Res. (1998). doi:10.1016/S0022-3956(98)00033-8 
38. Urrila, A. S. et al. Frontal Cortex Myo-Inositol Is Associated with Sleep and Depression in Adolescents: A Proton Magnetic Resonance Spectroscopy Study. Neuropsychobiology 75, 21-31 (2017).

39. Edgar, N. \& Sibille, E. A putative functional role for oligodendrocytes in mood regulation. Transl. Psychiatry 2, e109-e109 (2012).

40. Czéh, B. et al. Chronic Social Stress Inhibits Cell Proliferation in the Adult Medial Prefrontal Cortex: Hemispheric Asymmetry and Reversal by Fluoxetine Treatment. Neuropsychopharmacology 32, 1490-1503 (2007).

41. Banasr, M. et al. Chronic Unpredictable Stress Decreases Cell Proliferation in the Cerebral Cortex of the Adult Rat. Biol. Psychiatry 62, 496-504 (2007).

42. Hnilicová, P. et al. Altered hypothalamic metabolism in early multiple sclerosis - MR spectroscopy study. J. Neurol. Sci. 407, 116458 (2019).

43. Kantorová, E. et al. Hypothalamic damage in multiple sclerosis correlates with disease activity, disability, depression, and fatigue. Neurol. Res. 39, 323330 (2017).

44. Mayberg, H. S. et al. Deep Brain Stimulation for Treatment-Resistant Depression. Neuron 45, 651-660 (2005).

45. Kullmann, S. et al. Brain Insulin Resistance at the Crossroads of Metabolic and Cognitive Disorders in Humans. Physiol. Rev. 96, 1169-209 (2016).

46. Dimitriadis, G., Mitrou, P., Lambadiari, V., Maratou, E. \& Raptis, S. A. Insulin effects in muscle and adipose tissue. Diabetes Res. Clin. Pract. 93 , S52-S59 (2011).

47. Grillo, C. A. et al. Downregulation of hypothalamic insulin receptor expression elicits depressive-like behaviors in rats. Behav. Brain Res. 222, 230-5 (2011).

48. Benedict, C. et al. Intranasal insulin improves memory in humans. Psychoneuroendocrinology 29, 1326-34 (2004).

49. Lizarbe, B., Soares, A. F., Larsson, S. \& Duarte, J. M. N. Neurochemical Modifications in the Hippocampus, Cortex and Hypothalamus of Mice Exposed to Long-Term High-Fat Diet. Front. Neurosci. 12, 1-15 (2019).

50. Lizarbe, B. et al. Hypothalamic metabolic compartmentation during appetite regulation as revealed by magnetic resonance imaging and spectroscopy methods. Front. Neuroenergetics 5, 1-14 (2013).

51. Lizarbe, B., Campillo, B., Guadilla, I., López-Larrubia, P. \& Cerdán, S. Magnetic resonance assessment of the cerebral alterations associated with obesity development. J. Cereb. Blood Flow Metab. 40, 2135-2151 (2020).

52. Härter, M., Berger, M., Schneider, F. \& Ollenschläger, G. S3 Praxisleitlinien in Psychiatrie und Psychotherapie Nationale VersorgungsLeitlinie Unipolare Depression. Nervenheilkunde(2010).

53. Robert Koch Institut. RKI - Gesundheit A-Z - Robert Koch-Institut zum Weltgesundheitstag 2017: Daten und Fakten zu Depressionen. (2017). Available at: https://www.rki.de/DE/Content/Gesundheitsmonitoring/Gesundheitsberichterstattung/GesundAZ/Content/D/Depression/Daten_Fakten/daten_fakten_de (Accessed: 28th November 2019)

\section{Figures}

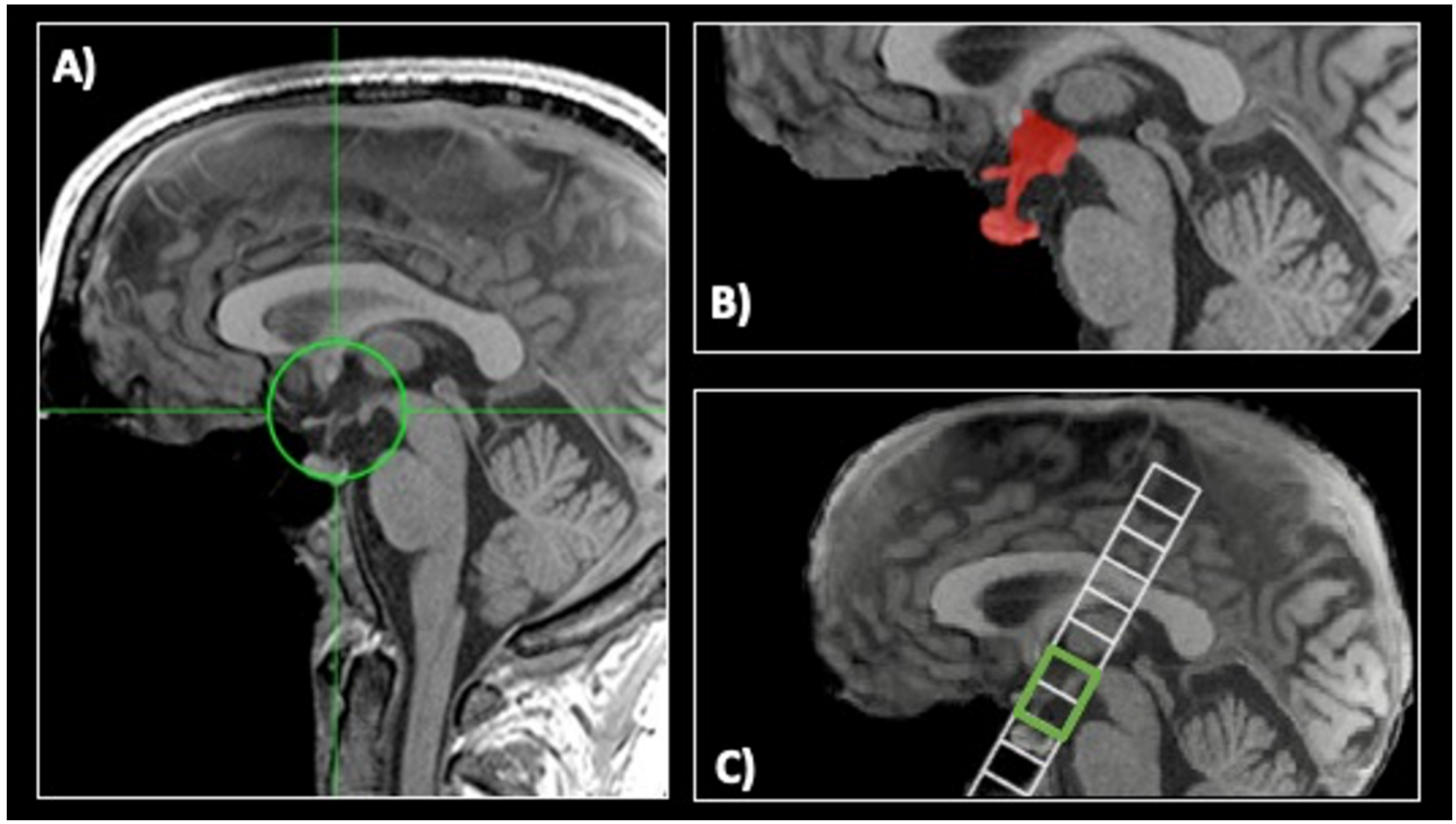

Figure 1 
(A) Localization of the hypothalamus in the MRI T1-MPRAGE sequence. (B) Marked region of interest (hypothalamus), including pituitary gland. (C). Diagonal localizer for the chemical shift imaging that included the region of interest - hypothalamus - in green. Pictures 1A and 1B were edited by using Horos v. 3.3.6 (The Horos Project \& OsiriX Team, United States of America). Picture 1C was obtained from the software TARQUIN v. 4.3.1. (Martin Wilson, Greg Reynolds,

United Kingdom)

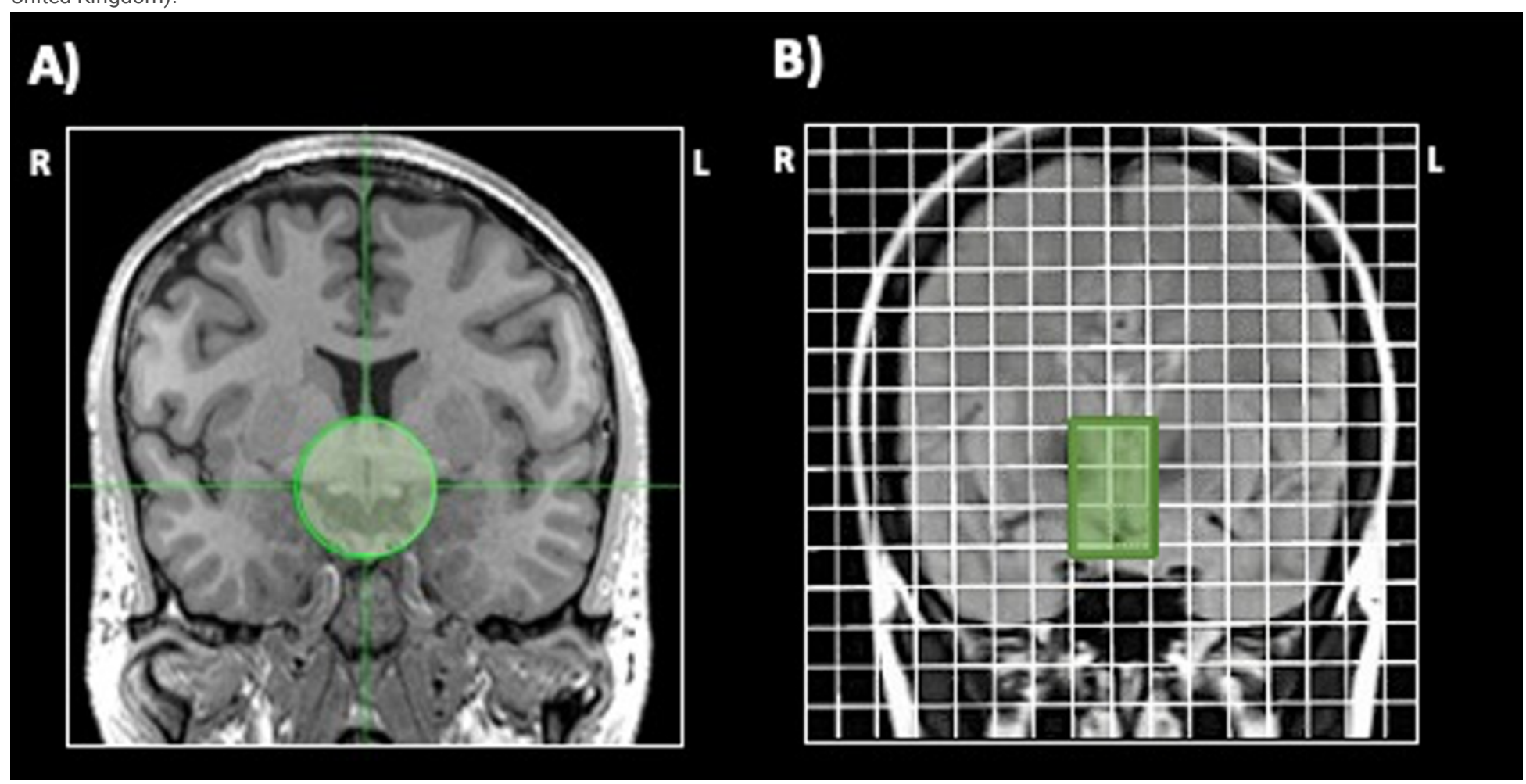

Figure 2

(A) Region-of-Interest (ROI) of the present study from a coronal slice. (B) CSI-MRS localizer - A grid plane from a diagonal slice representing the chemical shift imaging voxels, which include the hypothalamic region (green). Picture 2A was edited by using Horos v. 3.3.6 (The Horos Project \& OsiriX Team, United States of America). Picture 2B was obtained from the software TARQUIN v. 4.3.1. (Martin Wilson, Greg Reynolds, United Kingdom). 


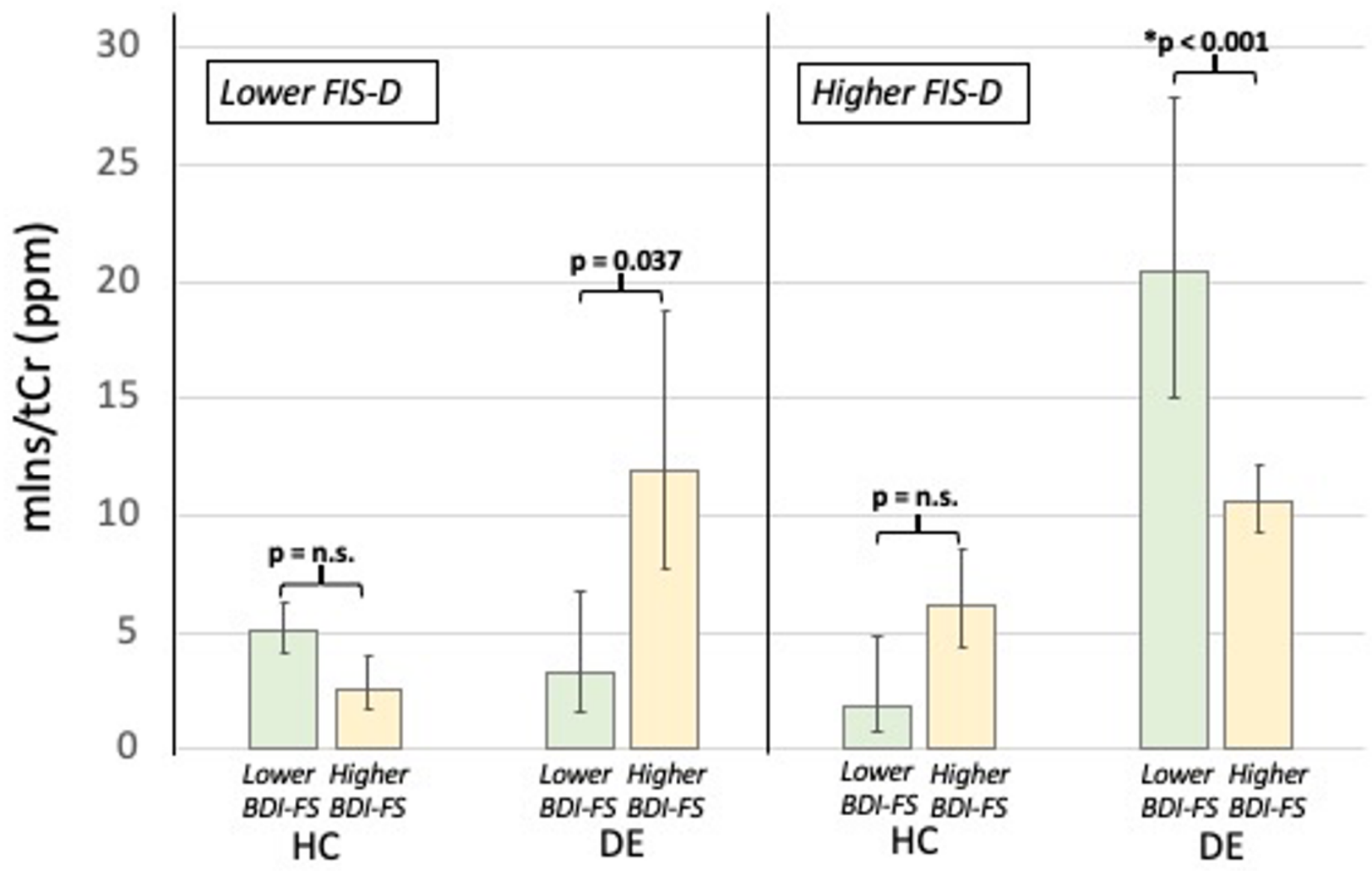

Figure 3

Graphical information from the Poisson regression. The figure represents information about hypothalamic mIns-concentration vs. Group status, BDI-FS and FIS-D values (Status x BDI-FS x FIS-D). P-values were marked whenever they overcome the corrected Bonferroni $p$-value threshold (PBONF $\leq 0.025)$. 


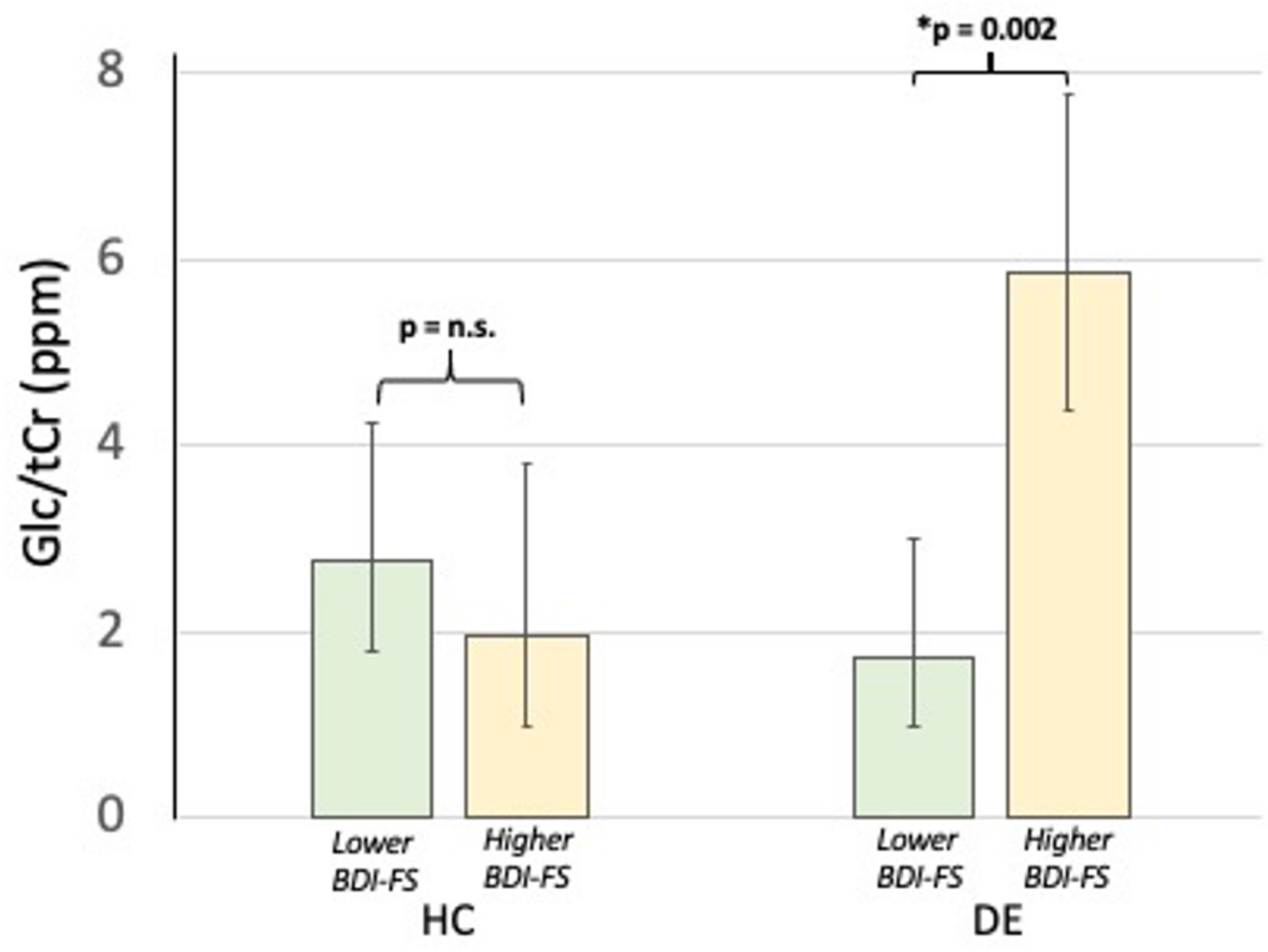

Figure 4

Graphical information from the Poisson regression. The figure represents information about hypothalamic Glc-concentration vs. Group status and BDI-FS values (Status x BDI-FS). P-values were marked whenever they overcome the corrected Bonferroni p-value threshold (PBONF $\leq 0.025)$. 